\title{
1 Dietary nutrient balance shapes phenotypic traits of Drosophila melanogaster in interaction with gut microbiota
}

3

\author{
Y. Henry ${ }^{1,2 *}$ J. Overgaard ${ }^{3}$, H. Colinet ${ }^{1}$
}

${ }^{1}$ ECOBIO - UMR 6553, Univ Rennes 1, CNRS, Rennes, France

${ }^{2}$ Eawag - Swiss Federal Institute of Aquatic Science and Technology, Dübendorf, Switzerland

${ }^{3}$ Department of Bioscience - Zoophysiology, University of Aarhus, Aarhus, Denmark.

*Corresponding author: Youn Henry - Eawag, Überlandstrasse 133, 8600 Dübendorf, Switzerland

Email: youn.henry@eawag.ch

\section{Abstract}

The dietary nutrient composition can affect insects' phenotypes by modulating their physiology. Furthermore, diet can affect gut microbiota composition and abundance, with indirect consequences for the host. In this study, we reared Drosophila melanogaster on five different diets; three with balanced sugar:yeast ratio, but with increasing caloric content $(2: 2,8: 8,16: 16$, in weight $\%)$, and two with imbalanced sugar:yeast ratio, either with low sugar and high yeast content (2:16) or vice-versa (16:2). In each of these diets, we compared flies with conventional vs. artificially altered gut microbiota with antibiotics that reduced the bacterial load. The antibiotic treatment also had the surprising effect of increasing the amount of live yeast associated with the flies. We characterized flies from these ten treatments ( 5 diets x 2 microbiota) in terms of development, body mass, food preference, body reserves, metabolic rate and a range of stress tolerance traits (heat, cold, starvation and desiccation tolerance). Diets, and to a lesser extent antibiotic treatment, affected development rate, weight, and cold tolerance of adult flies. Other traits such as energy reserves, metabolic rate, food preference, or starvation tolerance were affected by diet alone. When detected, the effect of antibiotic treatment was stronger in yeast-poor diets, suggesting that gut bacterial community might help to counterbalance nutritional deficiencies. These results show that changes in dietary factors lead to a global re-organization of fly's physiology and development while the manipulation of gut microorganisms had minor effects that were mainly seen in case of protein restriction.

Keywords: nutrition; microbiota; developmental plasticity; stress tolerance; metabolic rate

Short title: Diet and microbiota shape phenotypes

This document is the accepted manuscript version of the following article: Henry, Y., Overgaard, J., \& Colinet, H. (2020). Dietary nutrient balance shapes phenotypic traits of Drosophila melanogaster in interaction with gut microbiota. Comparative Biochemistry and Physiology Part A: Molecular and Integrative Physiology, 241, 110626 (10 pp.). https://doi.org/10.1016/j.cbpa.2019.110626 


\section{Highlights}

- We expected interactions between diet balance and microbiota on phenotypes.

- The physiological impact of microbiota was limited in comparison with the one of diets.

- Yeast content in food drove most nutritional effects on development and metabolism.

- Sugar-rich diets improved cold and starvation tolerance. 
Dietary Sugar:Yeast ratio

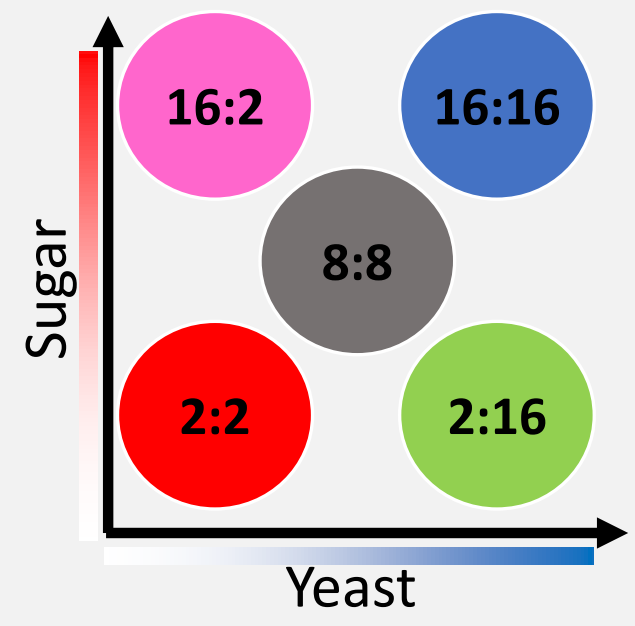

Phenotype

Body composition

Development

Metabolism

Behaviour

Stress tolerance 


\section{Introduction}

Most animals have a preferred dietary target in terms of macronutrient composition and amount (Simpson and Raubenheimer, 2012). In insects, the inability to meet this nutritional target represents a great challenge as nutrition affects the physiology and performance of individuals, including reproduction, development, and lifespan (Lee et al., 2008; Matzkin et al., 2011). Numerous studies addressed nutritional questions focusing on caloric restriction (Bartke et al., 2001; Masoro, 2003; Rogina et al., 2002). Food shortage may result in energy trade-off that manifests as reduced or delayed investment in development and reproduction (Edgar, 2006; Koyama et al., 2013). The shift from reproduction to survival mode is assumed to be a way to cope with harsh environment by increasing lifespan and promoting stress resistance, at the cost of a slow development (Burger et al., 2007; Carvalho et al., 2012; Kolss et al., 2009; Rion and Kawecki, 2007). Any change in nutritional supply can have considerable consequences for ecologically relevant traits of species (Raubenheimer et al., 2009), and these changes may also be driven by nutritional balance (sugar:protein ratio), independent of the caloric content (Fanson et al., 2009; Mair et al., 2005; Solon-Biet et al., 2014). Experiments using artificial food with controlled nutrient compositions have helped to disentangle the contributions of specific macronutrients on insects' phenotype. For example, the "geometric framework" represents a robust method to analyze the consequences of protein/carbohydrate ratios (Lee et al., 2008; Simpson and Raubenheimer, 2012) and studies have demonstrated how low protein:carbohydrate ratios reduce development rate but increase lifespan across diverse insect species (Fanson et al., 2009; Lee et al., 2008; Simpson and Raubenheimer, 2012; Skorupa et al., 2008). Insects display various strategies to counterbalance nutritional deficiencies. Behavioral shifts represent a first response to dietary pressures, whether they manifested in food preference changes, or increased foraging (e.g. Corrales-Carvajal et al., 2016; Simpson et al., 2006). Insects may also deal with nutritional imbalance through internal physiological adjustments that can help to maintain nutritional homeostasis. For instance, nutrient sensing in the gut is regulating molecular signaling pathways such as IIS/TOR (for insulin-like growth factor signaling/target of rapamycin) that are responsible for growth (Kapahi et al., 2004; Koyama et al., 2013; Layalle et al., 2008).

Mutualistic relationships with microorganisms may provide advantageous nutritional functions to insects, including the degradation and detoxification of indigestible food and synthesis of essential nutrients (Douglas, 2009). Understanding the complex tripartite interaction among diet, microbiota and host traits represents an exciting and novel challenge in the field of nutritional ecology (Jehrke et al., 2018). In Drosophila flies, some bacterial taxa like Lactobacillus plantarum or Acetobacter sp can promote growth through the activation of insulin pathways and partially compensate for detrimental effects of protein-poor nutrition (Matos et al., 2017; Shin et al., 2011; Storelli et al., 2011). Commensal bacteria have also been found to influence behavioral decisions by limiting yeast appetite and buffer for 
specific amino-acid depletion (Leitão-Gonçalves et al., 2017). Furthermore, the gut microbiota can directly contribute to the nutrient supply of D. melanogaster, and thereby affect both lipid and carbohydrate metabolism (Ridley et al., 2012; Wong et al., 2014). Other roles of gut microbiota include improved peptidase activity (Erkosar et al., 2015) and provision of secondary metabolites, vitamins, and amino acids (Sannino et al., 2018; Yamada et al., 2015).

The aforementioned studies suggest that the presence of a functional microbiota could promote the host's nutritional balance and fitness. However, benefits are likely to vary according to diet, bacterial taxa, as well as host genotype (Dobson et al., 2015; Newell and Douglas, 2014; Wong et al., 2014). For instance, in $D$. suzukii, the presence of a functional microbiota was found to be mandatory for survival on specific poor diets, but deleterious for lifespan on balanced diets (Bing et al., 2018). Likewise, multiple studies have also reported links between stress tolerance and microbiota in insects (Ferguson et al., 2018; Henry and Colinet, 2018; Moghadam et al., 2018; Montllor et al., 2002; Russell and Moran, 2006). Since nutritional composition and microbiota may both affect insect phenotypes, including stress tolerance, it is of interest to examine how these factors compare and if one factor is more dominant than the other. As an example, it is known that carbohydrate and lipid reserves vary according to nutrition (Lee and Jang, 2014; Wong et al., 2014) but also according to gut microbiota (Huang and Douglas, 2015; Ridley et al., 2012; Wong et al., 2014), and that these reserves are important for tolerance to thermal, desiccation, and starvation stress (Arrese and Soulages, 2010; Ballard et al., 2008; Colinet et al., 2013; Colinet and Renault, 2014; Klepsatel et al., 2016). Similarly, nutritional scarcity or altered gut microbiota can both increase development duration leading to hardened phenotypes that are better able to tolerate stress (Kolss et al., 2009; Storelli et al., 2011). To our knowledge there are only a handful of studies that have investigated the impact of nutritional variables in insects harboring contrasted gut microbiota compositions or abundances (Chaston et al., 2016; Huang and Douglas, 2015; Ridley et al., 2012; Wong et al., 2014).

Here, we tested i) the effect of dietary restriction using balanced diet and ii) the effect of isocaloric but imbalanced diets with skewed sugar:yeast ratios (S:Y), on D. melanogaster. These dietary manipulations were combined (or not) with an antibiotic treatment altering the gut microbiota. This experiment aimed to disentangle the role microorganisms in the host-nutrition interaction. We first hypothesized that moderate nutrient scarcity of particular proteins (here added as yeast in diets) would decrease development rate, body weight, and metabolic rate, but provide larger energy reserves and in fine higher stress tolerance. In addition, we expected that flies could be able to show preferences for specific nutrient in two-choices experiments, with a more marked preference for the limiting nutrient in imbalanced diets. Knowing that gut microorganisms may provide nutrients for their host, we also predicted that microbial depletion would amplify phenotypical and behavioral responses in poor nutritional situations, including low-calorie diets but also imbalanced diets. As a result, we expected the 
importance of microbiota depletion to vary according to diet: on rich diets, microbiota alteration could be neutral or beneficial, whereas it could strengthen the nutritional stress on poor diets.

\section{Methods}

\section{Fly stocks and culture medium}

We conducted the experiments on an outbred laboratory population of Drosophila melanogaster derived from wild individuals collected in September 2015 in Brittany (France). Fly stocks were maintained at $25^{\circ} \mathrm{C}$ under a 12:12 L:D photoperiod, on standard fly medium comprising $80 \mathrm{~g}$. $\mathrm{L}^{-1}$ of inactive brewer yeast (MP Biochemicals 0290331205), 50 g. L-1 of sucrose, $10 \mathrm{~g} . \mathrm{L}^{-1}$ of agar (SigmaAldrich A1296), supplemented with $8 \mathrm{~mL} . \mathrm{L}^{-1}$ of $10 \%$ methyl 4-hydroxybenzoate (Sigma-Aldrich H5501). Wolbachia symbiont was absent from the population.

\section{Dietary and microbiota manipulations}

Five diets comprising different amounts of sugar and yeast were tested. The experimental design was adapted from Zhu et al. (2014) (see fig. S1). The following S:Y ratios were used: 2:2, 8:8, 16:16, 2:16 and 16:2 (amounts expressed in weight \%). 2:2, 8:8, and 16:16 represented balanced diets with gradual increase in the caloric content, whereas 2:16 and 16:2 represented isocaloric diets (Min et al., 2007) but with imbalanced compositions (poor in sugar and rich in yeast, and vice-versa). Except for sugar and yeast concentrations, diets were prepared similarly to standard fly medium, and $40 \mathrm{~mL}$ of food was poured per $230 \mathrm{~mL}$ plastic bottle.

For each tested $\mathrm{S}: \mathrm{Y}$ ratio, we compared flies with intact microbiota to flies with altered microbiota, resulting in a total of 10 treatments (see fig. S1). The five diet treatments with microbiota alteration are indicated with single quotes (S:Y ratios: 2:2', 8:8', 16:16', 2:16' and 16:2'). Microbiota alteration was obtained by egg dechorionation using successive two minutes wash in $2.7 \%$ hypochlorite and two minutes in $70 \%$ ethanol, followed by three rinses in sterile miliQ water. In addition, dechorionated eggs were placed on food supplemented with an antibiotic cocktail: $50 \mu \mathrm{g}$ ampicillin (Sigma-Aldrich A9518), $50 \mu \mathrm{g}$ kanamycin (Sigma-Aldrich K4000), $50 \mu \mathrm{g}$ tetracyclin (Sigma-Aldrich T7660) and $15 \mu \mathrm{g}$ erythromycin (Sigma-Aldrich E5389) per liter of fly food (Téfit et al., 2018). Eggs for the untreated condition were not washed at all, as a simple water wash may affect bacterial abundance on the chorion. For simplicity, we use the term "antibiotic treatment" in the manuscript to refer to this microbiota alteration procedure including dechorionation and antibiotics. We roughly counted eggs to get approx. 500 eggs per bottle, hence avoiding crowding (Henry et al., 2018). After emergence, we changed bottles for new ones every second day. 


\section{Bacterial and yeast abundance measurement}

We counted colony forming units ( $\mathrm{CFU}$ ) on MRS media to estimate microorganisms load in flies' gut and control the efficiency of the antibiotic treatment with a standard method adapted from Koyle et al. (2016). See ESM for detailed methods.

\section{Mass, water content, and total lipid content measurement}

Mated females (5-d old) from all treatments were snap frozen in liquid nitrogen. Five to seven pools of $\mathrm{ca} 20$ flies per treatment were randomly picked and then weighed using a microbalance. Samples were dried for $48 \mathrm{~h}$ at $60^{\circ} \mathrm{C}$ and re-weighed to determine their dry mass. Finally, samples were washed repeatedly in boiling petroleum ether for $72 \mathrm{~h}$ in order to remove all lipids, and re-dried to determine lean mass.

\section{Glucose and glycogen assays}

Glucose and glycogen measurements were performed using a colorimetric method adapted from Tenessen et al. (Tennessen et al., 2014). Six replicates, each consisting of pools of five 5-d old mated females were used. Flies were snap frozen in liquid nitrogen, dried at $60^{\circ} \mathrm{C}$ for $48 \mathrm{~h}$, and weighed using a microbalance. Flies were crushed in $500 \mu \mathrm{L}$ of PBS using a bead biting apparatus (TissueLyzer LT, Qiagen, Hilden, Germany). Enzymes in samples were heat-inactivated by a $10 \mathrm{~min}$ incubation at $70{ }^{\circ} \mathrm{C}$. Thirty microliters of these samples were dispensed in duplicates in a 96-well plate. In the first well of each duplicate, $100 \mu \mathrm{L}$ of GO reagent (Sigma Aldrich GAGO-20) were added, and in the second well, $100 \mu \mathrm{L}$ of GO reagent plus $0.1 \%$ amyloglucosidase (Sigma Aldrich 1602) were added. After 60 min incubation at $37{ }^{\circ} \mathrm{C}, 100 \mu \mathrm{L}$ of $12 \mathrm{~N}$ sulfuric acid was dispensed in all wells to stop the reaction. Absorbance was measured at $540 \mathrm{~nm}$. Glycogen concentration for each sample was determined by subtracting samples without aminoglucosidase (corresponding to glucose concentration) from samples with aminoglucosidase (corresponding to glucose plus glycogen concentration). Quantification was based on glucose and glycogen standard curves. See ESM for detailed methods.

\section{Development time}

Adult flies were allowed to lay eggs on standard medium for $8 \mathrm{~h}$ before being discarded. Eggs were then collected using a paint brush, treated with the dechorionation treatment or left untouched. Eggs were then placed on the surface of the food, in controlled density of 25 eggs per $40 \mathrm{~mL}$ vial filled with $5 \mathrm{~mL}$ of medium ( $\mathrm{N}=4$ vials per treatment). The end of the oviposition period was considered as the $t=0$ for the development of flies. Pupation and emergence events were checked twice a day.

\section{Metabolic rate assay}

Metabolic rate of the flies was indirectly estimated by the rate of $\mathrm{CO}_{2}$ production $\left(\mathrm{VCO}_{2}\right)$ and $\mathrm{O}_{2}$ consumption $\left(\dot{\mathrm{VO}}_{2}\right)$ using repeated measurements of stop-flow respirometry (Lighton and Halsey, 2011). Here we used an experimental setup similar to that described by Jensen et al. (Jensen et al., 2014). 
cylindrical glass chambers (dimensions: D: $20 \mathrm{~mm} \mathrm{L:} 70 \mathrm{~mm}, 11-21$ individuals in each chamber). Within each chamber, flies had access to a piece of filter paper $(15 \mathrm{~mm} \times 15 \mathrm{~mm})$ with a droplet of the appropriate food to avoid starvation and desiccation. Chambers without flies, with or without filter paper and the droplet of food were also tested to correct measurements from background fluctuations. Two parallel 8-channels-multiplexers (RM Gas Flow Multiplexer, Sable Systems, Las Vegas, NV, USA) controlled the sequentially flushing and closing of the chambers such that the stop-flow respirometry system enabled us to obtain repeated measures of $\dot{\mathrm{VCO}}_{2}$ and $\dot{\mathrm{V}}_{2}$ over $24 \mathrm{~h}$. Measurements were performed independently at two experimental temperatures by placing the metabolic chambers in a climate chamber maintained at $15{ }^{\circ} \mathrm{C}$ or $25^{\circ} \mathrm{C}(12: 12 \mathrm{~L}: \mathrm{D})$. The low temperature ensured measurements at which locomotor activity is much reduced, allowing to infer the basal levels of gas exchange.

At the end of the experiment, flies were collected, killed by placing them briefly at $-20^{\circ} \mathrm{C}$ after which they were weighed using a microbalance (Sartorius Laboratory Balance type 1712, Göttingen, Germany). Analysis of respiratory data and estimation of basal metabolic rate were performed as in Jensen et al. (2014). See ESM for detailed methods.

\section{CAFE assay}

Mated female flies from all treatment combinations were used to measure feeding rates and preferences using capillary feeding method (CAFE) adapted from (Ja et al., 2007). Flies were left in empty vials for one hour before starting the experiments. Next, seven individuals were placed in $40 \mathrm{~mL}$ vials with access to food via $2 * 210 \mu \mathrm{L}$ glass capillaries, containing either $8 \%$ sucrose or $8 \%$ autoclaved yeast solutions ( $\mathrm{N}=5-8$ vials per treatment). These foods were randomly supplemented with blue and red commercial food coloring (Vahiné, France) as this improved precision of food intake measurements (Diegelmann et al., 2017). Vials were placed in a hermetic tank with humidified towels to limit evaporation $\left(25^{\circ} \mathrm{C}, 12: 12 \mathrm{~L}: \mathrm{D}\right)$. Eight vials with capillaries but without flies were used to control for evaporation. Food consumption was measured using an electronic caliper from the reduction in "liquid height" in the capillaries after $10 \mathrm{~h}$.

\section{Thermal stress assays}

Adult flies from all the diets*microbiota combinations were sexed under light $\mathrm{CO}_{2}$ anesthesia ( $<2 \mathrm{~min}$ ), allowed to recover at least $48 \mathrm{~h}$, and 5-7-d mated females were used to assess thermal stress tolerance.

For acute stress survival, 180 flies per treatment were placed into $50 \mathrm{~mL}$ vials and immersed in a thermoregulated bath filled with a glycol-water mix set at $-3.5^{\circ} \mathrm{C}$ or $38.5^{\circ} \mathrm{C}\left( \pm 0.1^{\circ} \mathrm{C}\right)$ for cold and heat stress respectively. From $t=0$ to $t=120 \mathrm{~min}, 20$ individuals per treatment were removed from the bath each $15 \mathrm{~min}$ and transferred into fresh vials with standard food medium to recover at $25^{\circ} \mathrm{C}$. Survival 
For critical minimal and maximal temperatures $\left(\mathrm{CT}_{\min }\right.$ and $\left.\mathrm{CT}_{\max }\right), 25$ mated females per treatment were individually placed in 5-mL glass vials immersed in a thermoregulated glycol-water bath set at $20{ }^{\circ} \mathrm{C}$. Next, flies were exposed to a slow ramping down or up at the rate of $\pm 0.1^{\circ} \mathrm{C} \mathrm{min}^{-1}$ and the temperature at which all movement ceased was recorded as the upper or lower thermal limit for a given individual.

\section{Starvation and desiccation assays}

Adult flies from all the diets*microbiota combinations were sexed under light $\mathrm{CO}_{2}$ anesthesia, allowed to recover at least $48 \mathrm{~h}$, and 5-7 d mated females were used to measure starvation tolerance. Twenty-five flies were individually placed in $40 \mathrm{~mL}$ vials filled with $5 \mathrm{~mL}$ of $1,5 \%$ agar-water medium to avoid desiccation $\left(25^{\circ} \mathrm{C}, 12: 12 \mathrm{~L}: \mathrm{D}\right)$. Survival was checked each two hours for the first $48 \mathrm{~h}$, and each four hours afterwards, until $100 \%$ mortality was reached. The time at which all movement ceased was recorded as survival time.

For desiccation, 25 mated females per treatment were individually placed in 5-mL glass vials closed with fine mesh to allow air circulation. These vials were then placed in a hermetic glass tank with a four-centimeter layer of silica-gel that ensured a low air humidity $\left(<5 \% \mathrm{RH}, 25^{\circ} \mathrm{C}, 12: 12 \mathrm{~L}: \mathrm{D}\right)$. Survival of flies was checked each hour for $24 \mathrm{~h}$. The time at which all movement ceased was recorded as survival time.

\section{Statistical analyses}

All statistical analyses were performed with R (R Core Team, 2017). CFU counting was analyzed using negative binomial regression accounting for replicates random effect. Survival was analyzed using cox models for tolerance to starvation and desiccation, that were both measured over time with repeated observations. Pairwise differences were assessed using log-rank Mantel-Cox test with Benjamini-Hochberg correction for multiple comparisons. Acute stress tolerance (cold and heat) were analyzed using binomial GLM with logit link function (binomial survival $\sim \operatorname{diet} *$ microbiota*exposure duration) followed by Tukey HSD post-hoc test. ANOVA were used for all other metrics, (metric diet*microbiota), followed by Tukey HSD post-hoc test. The part of the variance attributed to diet, microbiota, and their interaction was extracted using 'relaimpo' package (Groemping, 2006). For all tests, significance threshold was fixed at $\mathrm{p}<0.01$.

\section{Results}

\section{Bacterial and yeast load}

The antibiotic treatment used in the present study almost completely killed the microbial 230 community in the treated flies (fig. 1A; fig. S2). The bacterial load was already rather low in conventional flies from several diets, especially those with low yeast supply (2:2 and 16:2). Yeast colonization was also much dependent on diet and microbiota alteration treatment (fig. 1B; table 1). While the antibiotics markedly reduced bacterial load, yeasts were more abundant in bacteria-depleted 
flies. Some yeasts colonies were observed in conventional flies, but mostly in yeast-rich diets (16:16 and 2:16) (fig. 1B; fig. S2).

\section{Body composition}

Fresh mass was affected by dietary treatments such that yeast-poor diets (2:2 and 16:2) were significantly smaller (by about $40 \%$ ) in comparison with yeast-rich diets (fig. 2A; table 1). Balanced diets 8:8 and 16:16 did not show significant mass differences linked to caloric content (table S1). Even if the main effect of microbiota and the diet*microbiota interaction were significant, they only accounted for a small part of the mass total variance (table 1), and pairwise differences could not detect clear pattern linked to these two variables (table S1). Water content relative to mass was increased in sugarpoor diets (2:2 and 2:16) in comparison with others diets, but no significant effect of microbiota was observed (fig. 2B; table 1). Similarly, total lipid content was linked to the dietary sugar content and not to microbiota status (fig. 2C; table 1). Thus, flies from sugar-poor diets (2:2 and 2:16) had half the lipid content of flies from the others diets (fig. 2C; table S1). Carbohydrate reserves (glucose and glycogen) were highly influenced by diet (fig. 2D \& E; table 1). Flies that developed on diets with low yeast content (2:2 and 2:16) were characterized by high levels of glucose and glycogen compared to other treatments (table S1). Sugar-rich diets did not result in carbohydrate rich flies when the S:Y ratio was balanced (8:8 and 16:16). Diet*microbiota interactions were significant for both glucose and glycogen contents, accounting for about $10 \%$ of the total variance. This manifested in higher levels of carbohydrates in 2:2' compared to 2:2, but lower glucose level in 16:2' compared to 16:2 (fig. 2D \& E; table S1). Overall, yeast content of the food drove most of the body composition, including weight and carbohydrates reserves. Sugar content affected lipid reserves, and we did not find evidence to suggest that the antibiotics treatment affected this relationship.

\section{Development, metabolism and nutritional behavior}

Development time (fig. 3A) was significantly impaired by diet and diet*microbiota interaction (table 1). Diets with low yeast content (2:2 and especially 16:2) increased development time compared to the other treatments (pairwise comparisons in table S1). Antibiotic treatment also tended to delay development (fig. 3A) but here the effect was diet-specific with considerable delay of development in the sugar rich 16:2' diet but only minor delay in the others (fig. 3A; table S1). Metabolic rate, measured as oxygen consumption, was high in yeast-rich diets $(8: 8 ; 16: 16 ; 2: 16)$, and low in yeast-poor ones (fig. 3B; table S1). Caloric content of the diets seemed to have limited effect on oxygen consumption since the highly caloric but yeast-poor diet (16:2) showed a rather low oxygen consumption, not different from the 2:2 diet (table S1). There were no significant effects of microbiota on metabolic rate either (table 1). As expected, balanced diets $(2: 2 ; 8: 8 ; 16: 16)$ were characterized by an intermediate RQ (0.9$0.94)$, sugar-rich diets (16:2) had a RQ very close to 1 and yeast-based diets (2:16) had a RQ of 0.8-0.85 (fig. 3C; table S1). We could not evidence pairwise differences between the RQ of flies treated or not with antibiotics (table 1). Food preference varied between diets in a pattern that indicated compensation 
for lacking nutrient related to the rearing diet (fig. 3D; table 1). Sugar consumption was lower than yeast consumption in all diets except in the flies reared on yeast-rich diet (2:16). Conversely, flies from yeastpoor diets (2:2 and 16:2) were those most attracted by yeast (fig. 3D; table S1). Total food consumption also showed compensatory feeding as flies reared on reduced caloric content $(2: 2)$ fed more than all other dietary groups (fig. 3E). Food preference and total food consumption variations mostly resulted from diet composition, with no significant effect of microbiota (table 1). In conclusion, all developmental, metabolic, and behavioral effects were mainly linked to yeast amount in the food. The antibiotic treatment increased the developmental delay only in yeast-poor diets that already showed slow development.

\section{Stress tolerance}

Cold survival was significantly and mainly affected by diets (fig. 4A; table 1). Flies from balanced diets with medium or large caloric content (8:8 and 16:16) were sensitive to cold while flies from yeast-poor ones (2:2 and 16:2) were especially tolerant (fig. 4A; table S1). Significant effect of microbiota was detected on cold survival, as flies from antibiotics diets tended to survive longer exposures than conventional flies, but its impact was weak considering it only represented $3 \%$ of explained variance (table 1). Interestingly, we did not observe that differences in cold survival reflected in $\mathrm{CT}_{\text {min }}$ differences (fig. 4B). Although there was a significant effect of diet, it only accounted for a limited part of the explained variance (fig. $4 \mathrm{~B}$; table 1 ). $\mathrm{CT}_{\min }$ was mainly affected by the microbiota alteration and the diet*microbiota interaction (table 1). Except for the 2:16 diet, flies treated with antibiotics entered chill coma at lower temperatures than flies with intact microbial communities (fig. 4B; table S1). Heat tolerance (acute \& knockdown) was almost unaffected by diet and microbiota (fig. 4C \& D; table 1). Diet, microbiota, and diet*microbiota interaction all affected starvation tolerance (fig. 4E; table 1). Survival to starvation was greatly enhanced in 16:2 flies, reduced in 2:16, and intermediate in all balanced diets $(2: 2,8: 8,16: 16)$ (fig. 4E; table $\mathrm{S} 1)$. The antibiotic treatment globally reduced the starvation tolerance (e.g. 16:16 vs 16:16'), although no pairwise differences were found significant (table 1; table S1). For desiccation tolerance, we observed a small but significant effect of diet (fig. 4F; table 1). The different dietary groups varied by less than five hours in survival but this was sufficient to separate the most (16:16 and 8:8) from the least resistant flies (2:16) (fig. 4F; table S1). No specific combination of diet and antibiotic treatment lead to globally more tolerant individuals against all stressors. Antibiotics treatment either improved tolerance to cold or reduced starvation tolerance. Flies from yeast-poor diets tolerated cold and starvation better, but heat and desiccation tolerance remained almost unaffected.

\section{Discussion}

Although dietary effects have been broadly explored in Drosophila (Burger et al., 2007; Kristensen et al., 2016; Matzkin et al., 2011), it is not yet clear whether phenotypical changes result 
exclusively from dietary factors per se or whether they also entail some indirect influences from gut microbiota. In this study we investigated how both the caloric content of diets (sugar and yeast concentration) and the composition (sugar:yeast ratio) affected phenotypes of flies harboring conventional or altered microbiota.

\section{Dietary effects on nutritional phenotype}

Differences in diets were clearly responsible for the largest part of observed phenotypes, representing 80-99\% of the explained variance in almost all of the measured traits (table 1). A key result is that dietary deprivation or supplementation of one specific nutrient (carbohydrates or proteins) often lead to similar responses as global caloric deprivation or supplementation of both nutrients. This is consistent with the idea introduced by Mair et al. (2005), and followed by many studies using the geometric framework (Lee et al., 2008; Simpson and Raubenheimer, 2012), that nutritional balance is the main limiting nutritional factor. Yeast content generally drove organisms to the extreme phenotypes, irrespective of the sugar content. Yeast-poor diets generated leaner individuals, displaying only half the size of most other developmental groups, and with relative high glucose and glycogen content (fig. 2A, $\mathrm{D}$, E). This result is consistent with previous studies in which protein deprivation switched the metabolism to carbohydrates-based reserves (Simmons and Bradley, 1997; Wong et al., 2014). We noticed flies being offered a choice between two nutrients would try to compensate for the yeast scarcity via increased preference for yeast over sugar, and global increased hunger (fig. 3D, E). Such compensatory feeding behavior probably indicates that flies reared on imbalanced diets suffered from malnutrition, and were unable to meet their target nutrient supply in a food where everything is mixed (Fanson et al., 2012; Leitão-Gonçalves et al., 2017). We observed slow development at the larval stage and a low metabolic rate in flies reared on yeast-poor diets (fig. 3A, B). These results contrast with the metabolic rate stability previously observed in individuals subjected to dietary restriction at the adult stage (Hulbert et al., 2004). However, in our experimental setup, we performed dietary manipulations for the entire larval development. This demonstrates that nutrition may deeply affect physiology of individuals during their early development, and carry over to the adult stage, as it has also been found for many other phenotypic traits (De Block and Stoks, 2008; Gandolfi et al., 2003; Hahn, 2005; Hoover et al., 2005). Sugar content in food also influenced flies' phenotypes, though less obviously than yeast.

High amounts of sugar increased total lipid content and decreased water content in flies, as previously observed (fig. 2B, C) (Jehrke et al., 2018; Matzkin et al., 2011; Musselman et al., 2011; Wong et al., 2014). In addition, yeast-poor and sugar-rich diet (16:2) prolonged the developmental time, as compared to the low caloric but balanced diet (2:2). This is in agreement with the results of Musselman et al. (2011) who reported that high-sucrose feeding resulted in a significant developmental delay. Indeed, excessive dietary sugar tends to suppress protein storage in Drosophila (Skorupa et al., 2008). It is possible that the high sugar content generated energetic satiety before the protein supply target was met. 
Such a response would indirectly reduce the uptake of proteins (as dietary yeast) and slow development on a diet that is rich in carbohydrate but limited in protein (Lee et al., 2008).

\section{Microbiota effects on nutritional phenotype}

Combining eggs dechorionation and antibiotics successfully reduced gut bacterial load, but lead to an unexpected accumulation of gut yeasts (fig. 1A, B). Therefore, instead of comparing conventional vs. axenic flies, we rather compared flies with intact microbiota (high gut bacteria load, low gut yeast load) to flies with manipulated microbiota (low gut bacteria load, high gut yeast load). As yeast are a known source of dietary protein and micronutrients (Keebaugh et al., 2018), it is possible that the phenotypes resulted either from the lack of gut bacteria or from the increased abundance of gut yeasts. This prevent us from drawing unequivocal conclusions about microbiota effect as a whole, and we therefore limit our discussion to the effect of antibiotic treatment in general.

Bacteria and yeast abundances in the gut were positively correlated with yeast concentration in the food, as was also found by Galenza et al. (2016) and Keebaugh et al. (2018). We only observed contrasted microbiota load and compositions in the yeast-rich diets $(8: 8,16: 16,2: 16)$, resulting in strong diet-dependency of most phenotypic traits affected by the antibiotic treatment. Our procedure did not provide the desired control over the microbiota, but we still observed phenotypic effects that were consistent with the literature. In line with findings from Wong et al. (2014b) we observed a slight increase of mass in antibiotic treated flies from low calorie diets (2:2') and slight decrease when they were reared on energy rich diets (16:16') (fig: 2A). Unlike in vertebrate models, axenic flies generally show hyperlipidemia when reared on poor diets (Wong et al., 2014). This is possibly because axenic individuals miss bacteria that usually consume dietary sugars in place of their host (Bäckhed et al., 2004). In the present study, we did not observe such response. Here, the antibiotic treatment did not change the lipid content under the poorest nutritional conditions (2:2) (fig. 2C-E), which is consistent with the findings of Ridley et al. (2012). Interestingly, antibiotics increased the carbohydrate content (glucose and glycogen) of flies reared on poor diets (2:2) and conversely reduced glucose content of flies reared under sugar-rich conditions (16:2). We did not detect any significant impact of the antibiotic treatment on oxygen consumption or RQ, whatever the diet (fig. 3C). This is surprising because one may expect changes in carbohydrates reserves to affect the available energy fuel and consequently to trigger metabolic rate changes as observed by Ridley et al. (2012). One possible explanation is that the effect of diet differences outweighed a putative minor effect of antibiotics, especially given that flies had access to their specific food during measurements of metabolic rate.

Alteration of energy reserves by microbiota could result from many kinds of host-microbiota relationships, including changes in host's behavior and dietary preference (Douglas, 2018). However, we failed to identify any "buffering" effect of our antibiotic treatment on yeast appetite as it is described by Leitão-Gonçalves et al. (2017), even when the nutrient supply was low. It is possible that high live 
yeasts abundances in antibiotic-treated conditions represented a protein source and prevented us from observing such behaviors. The most striking effect of the antibiotic treatment was on development (fig. 3A). Developmental time increased for flies treated with antibiotic, especially in flies reared on food overloaded with sugar (from $+1 \mathrm{~d}$ in balanced diets $8: 8$ and 16:16 to $+4 \mathrm{~d}$ in 16:2), but without affecting the poorest diet $(2: 2)$. Whether the microbiota affected the supply of available sugars in the food by consuming them (Chaston et al., 2016; Huang and Douglas, 2015; Ridley et al., 2012), or changed flies' use of energy reserves acting on hormonal pathways (Ridley et al., 2012; Shin et al., 2011; Storelli et al., 2011) needs future investigations.

\section{Consequences of diet-microbiota interactions on stress tolerance}

Considering the role of microbiota in nutrition (Douglas, 2009) and the role of nutrition in stress tolerance (Andersen et al., 2010; Sisodia and Singh, 2012), we expected to observe marked responses in stress tolerance traits related to both diet and microbiota. We did not detect any effect of diet or antibiotic treatment on heat stress tolerance (fig. 4C, D). These results contrast with those of Andersen et al. (2010) and Sisodia and Singh (2012) who found that protein-rich diets improved heat stress tolerance, although diets and metrics used to assess tolerance are not directly comparable (Jørgensen et al., 2019). In contrast, resistance to cold stress was much more responsive to dietary conditions and manipulation of microbiota. Cold knockdown temperature showed rather small effect of diets (2:16 flies fell into coma at slightly lower temperatures than other diets), and a larger and consistent beneficial effect of the antibiotics treatment (fig. 4B). Conversely, acute cold stress survival was only weakly dependent on antibiotics treatment, but strongly dependent on nutrition, with low caloric content being beneficial for cold survival in comparison with rich diets (fig. 4A). The divergence between the two cold tolerance metrics is of interest because they rely on different physiological mechanisms: cold knockdown is linked to the loss of neurological function (mainly in the brain), and the survival after harsh cold exposure depends upon the ability to maintain ion balance (in the whole body) (Armstrong et al., 2012; Overgaard and MacMillan, 2017). There are energetic costs associated with ion balance regulation and one could speculate that dietary-induced changes in the metabolic rate and nutritional status affected the energy allocation and prevented the maintenance of ion homeostasis after harsh stress.

$$
\text { We observed a positive effect of dietary restriction on cold survival that diverges from previous findings }
$$
(Burger et al., 2007). Our restriction was more severe than that of Burger et al (2007) who used a restrictive diet comparable to our intermediate diet $(8: 8)$, which could explain this result. The lower knockdown temperature in yeast-rich and sugar-poor diet 2:16 diet is consistent with previous observations that excess in dietary sugar is not necessarily beneficial for cold tolerance (Colinet et al., 2013) and that yeasts, especially live ones, favor cold tolerance (Colinet and Renault, 2014; Le Bourg et al., 2015). Although increased cold tolerance of microbiota-altered flies is surprising (Henry and Colinet, 2018), it could be linked to the unexpected increased yeast gut content that resulted from our antibiotic treatment. The focus is often on bacteria as the main gut symbionts but the role of yeasts 
should not be neglected (Hoang et al., 2015). Live yeasts can affect Drosophila physiology and increase survival to infections and starvation, in addition to cold stress (Le Bourg et al., 2015; Le Rohellec and Le Bourg, 2009). The difference between the effects of dead and live yeasts suggests these microorganisms provide an active protection that goes beyond being a simple food source.

Starvation tolerance was improved in sugar rich diet particularly when dietary yeast was absent. This is not surprising since the sugar fed flies have a higher lipid content which has been shown to provide better tolerance to starvation in other Drosophila species (e.g. Ballard et al., 2008). Interestingly we did not find any effect of caloric restriction on starvation tolerance. Here, the lower metabolic rates likely offset the lower body mass of calorie-restricted individuals, leading to a neutral outcome. In addition, Burger et al. (2007) found that caloric restriction effects were time-dependent, and only turned detrimental after 20 days post-emergence. It is possible that we tested our individuals at an intermediate time, without marked trend for beneficial or detrimental effects. Antibiotic treatment had only little influence on starvation tolerance, which is coherent with its limited effect on body reserves.

Desiccation tolerance responded less than expected to dietary manipulation and antibiotics treatment. Both diet and microbiota have previously been shown to play a role in synthesis of cuticular lipids (Fedina et al., 2012; Heys et al., 2018), a family of molecules affecting water retention of insects (Gibbs et al., 1997). In addition, changes in metabolic rate between diets should also affect tolerance of flies since increased gas exchanges tend to increase dehydration (Gibbs et al., 2003). We observed little support for these effects in our results. At most, flies from diets with higher content in sugar were slightly better at dealing with dehydration stress than those with reduced amount of sugar in their diet. Total lipid content was higher in these flies, and this type of reserves is well suited for tolerance to hydric stress since lipids oxidation represent a decent source of water (Arrese and Soulages, 2010).

\section{Conclusions}

Overall, we showed that for most of the tested traits, diet was more determining than microbiota. The antibiotic treatment interacted with diets to alter several traits, but, except for $\mathrm{CT}_{\min }$, the strength of these effects was generally minor compared to the one of diets alone, encompassing over $80 \%$ of the explained variance (table 1). We failed to replicate the observation of hyperlipidemia, hyperglycemia and reduced metabolic rate previously detected in axenic flies (Wong et al., 2014). The absence of marked microbial effects could be related to our experimental procedure. Though antibiotics effectively eliminated bacteria, they also allowed yeasts to thrive even more than in conventional flies. Hence, it is unclear whether the relatively modest changes in phenotype associated with antibiotic treatments were a consequence of bacteria elimination or of yeast growth. Additionally, we cannot rule-out the importance of genetic background in our observations, possibly hampering our ability to replicate previous findings (Chaston et al., 2016). Regarding diet, we showed that its composition was often more important than its caloric content. Flies exposed to imbalanced diets with similar intermediate caloric 
contents exhibited divergent developmental responses, energy metabolism and stress tolerance. The combined restriction of sugar and yeasts often led to similar responses as yeast-deprived but sugar-rich diets, suggesting protein content (as dietary yeasts) was the main driver of observed changes in phenotype. Flies reared on yeast-poor diets, irrespective of the sugar content, were characterized by slow development, low body mass, large relative carbohydrates reserves, high metabolic rate and increased stress tolerance. Further investigations should aim to disentangle the role of live yeasts from the one of bacteria in various dietary conditions. This could give a finer understanding of these complex nutritional interactions and particularly improve our understanding of yeasts, both as members of the microbiota and as an important protein resource.

\section{Data accessibility}

Datasets are available in online repository https://doi.org/10.6084/m9.figshare.7594343

\section{Acknowledgements}

Authors kindly thank Heidi MacLean as well as Torsten Kristensen for suggestions and advices on methodological aspects (desiccation and CAFE experiments respectively), and Kirsten Kromand for very welcomed technical assistance.

\section{Funding}

This work was supported by a grant from the Danish Council of Independent Research (to J.O.) and the international mobility grant from Rennes Metropole (to Y.H.).

\section{References}

Andersen, L.H., Kristensen, T.N., Loeschcke, V., Toft, S., Mayntz, D., 2010. Protein and carbohydrate composition of larval food affects tolerance to thermal stress and desiccation in adult Drosophila melanogaster. Journal of Insect Physiology 56, 336-340. https://doi.org/10.1016/j.jinsphys.2009.11.006

Armstrong, G.A.B., Rodríguez, E.C., Meldrum Robertson, R., 2012. Cold hardening modulates K+ homeostasis in the brain of Drosophila melanogaster during chill coma. Journal of Insect Physiology 58, 1511-1516. https://doi.org/10.1016/j.jinsphys.2012.09.006

Arrese, E.L., Soulages, J.L., 2010. Insect Fat Body: Energy, Metabolism, and Regulation. Annual Review of Entomology 55, 207-225. https://doi.org/10.1146/annurev-ento-112408-085356

Bäckhed, F., Ding, H., Wang, T., Hooper, L.V., Koh, G.Y., Nagy, A., Semenkovich, C.F., Gordon, J.I., 2004. The gut microbiota as an environmental factor that regulates fat storage. PNAS 101, 15718-15723. https://doi.org/10.1073/pnas.0407076101

Ballard, J.W.O., Melvin, R.G., Simpson, S.J., 2008. Starvation resistance is positively correlated with body lipid proportion in five wild caught Drosophila simulans populations. Journal of Insect Physiology 54, 1371-1376. https://doi.org/10.1016/j.jinsphys.2008.07.009

Bartke, A., Wright, J.C., Mattison, J.A., Ingram, D.K., Miller, R.A., Roth, G.S., 2001. Extending the lifespan of long-lived mice. Nature 414, 412-412. https://doi.org/10.1038/35106646

Bing, X., Gerlach, J., Loeb, G., Buchon, N., 2018. Nutrient-Dependent Impact of Microbes on Drosophila suzukii Development. mBio 9, e02199-17. https://doi.org/10.1128/mBio.02199-17 
Burger, J.M.S., Hwangbo, D.S., Corby- Harris, V., Promislow, D.E.L., 2007. The functional costs and benefits of dietary restriction in Drosophila. Aging Cell 6, 63-71. https://doi.org/10.1111/j.1474-9726.2006.00261.x

Carvalho, M., Sampaio, J.L., Palm, W., Brankatschk, M., Eaton, S., Shevchenko, A., 2012. Effects of diet and development on the Drosophila lipidome. Molecular Systems Biology 8, 600. https://doi.org/10.1038/msb.2012.29

Chaston, J.M., Dobson, A.J., Newell, P.D., Douglas, A.E., 2016. Host Genetic Control of the Microbiota Mediates the Drosophila Nutritional Phenotype. Appl. Environ. Microbiol. 82, 671-679. https://doi.org/10.1128/AEM.03301-15

Colinet, H., Larvor, V., Bical, R., Renault, D., 2013. Dietary sugars affect cold tolerance of Drosophila melanogaster. Metabolomics 9, 608-622. https://doi.org/10.1007/s11306-012-0471-z

Colinet, H., Renault, D., 2014. Dietary live yeast alters metabolic profiles, protein biosynthesis and thermal stress tolerance of Drosophila melanogaster. Comp. Biochem. Physiol., Part A Mol. Integr. Physiol. 170, 6-14. https://doi.org/10.1016/j.cbpa.2014.01.004

Corrales-Carvajal, V.M., Faisal, A.A., Ribeiro, C., 2016. Internal states drive nutrient homeostasis by modulating exploration-exploitation trade-off. Elife 5. https://doi.org/10.7554/eLife.19920

De Block, M., Stoks, R., 2008. Short-term larval food stress and associated compensatory growth reduce adult immune function in a damselfly. Ecological Entomology 33, 796-801. https://doi.org/10.1111/j.1365-2311.2008.01024.x

Diegelmann, S., Jansen, A., Jois, S., Kastenholz, K., Escarcena, L.V., Strudthoff, N., Scholz, H., 2017. The CApillary FEeder Assay Measures Food Intake in Drosophila melanogaster. JoVE (Journal of Visualized Experiments) e55024. https://doi.org/10.3791/55024

Dobson, A.J., Chaston, J.M., Newell, P.D., Donahue, L., Hermann, S.L., Sannino, D.R., Westmiller, S., Wong, A.C.-N., Clark, A.G., Lazzaro, B.P., Douglas, A.E., 2015. Host genetic determinants of microbiota-dependent nutrition revealed by genome-wide analysis of Drosophila melanogaster. Nature Communications 6, 6312. https://doi.org/10.1038/ncomms7312

Douglas, A.E., 2018. The Drosophila model for microbiome research. Lab Animal 47, 157-164. https://doi.org/10.1038/s41684-018-0065-0

Douglas, A.E., 2009. The microbial dimension in insect nutritional ecology. Functional Ecology 23, 38 47. https://doi.org/10.1111/j.1365-2435.2008.01442.x

Edgar, B.A., 2006. How flies get their size: genetics meets physiology. Nat. Rev. Genet. 7, 907-916. https://doi.org/10.1038/nrg1989

Erkosar, B., Storelli, G., Mitchell, M., Bozonnet, L., Bozonnet, N., Leulier, F., 2015. Pathogen Virulence Impedes Mutualist-Mediated Enhancement of Host Juvenile Growth via Inhibition of Protein Digestion. Cell Host Microbe 18, 445-455. https://doi.org/10.1016/j.chom.2015.09.001

Fanson, B.G., Weldon, C.W., Pérez- Staples, D., Simpson, S.J., Taylor, P.W., 2009. Nutrients, not caloric restriction, extend lifespan in Queensland fruit flies (Bactrocera tryoni). Aging Cell 8, 514-523. https://doi.org/10.1111/j.1474-9726.2009.00497.x

Fanson, B.G., Yap, S., Taylor, P.W., 2012. Geometry of compensatory feeding and water consumption in Drosophila melanogaster. Journal of Experimental Biology 215, 766-773. https://doi.org/10.1242/jeb.066860

Fedina, T.Y., Kuo, T.-H., Dreisewerd, K., Dierick, H.A., Yew, J.Y., Pletcher, S.D., 2012. Dietary Effects on Cuticular Hydrocarbons and Sexual Attractiveness in Drosophila. PLOS ONE 7, e49799. https://doi.org/10.1371/journal.pone.0049799

Ferguson, L.V., Dhakal, P., Lebenzon, J.E., Heinrichs, D.E., Bucking, C., Sinclair, B.J., 2018. Seasonal shifts in the insect gut microbiome are concurrent with changes in cold tolerance and immunity. Functional Ecology 32, 2357-2368. https://doi.org/10.1111/1365-2435.13153

Galenza, A., Hutchinson, J., Campbell, S.D., Hazes, B., Foley, E., 2016. Glucose modulates Drosophila longevity and immunity independent of the microbiota. Biology Open 5, 165-173. https://doi.org/10.1242/bio.015016

Gandolfi, M., Mattiacci, L., Dorn, S., 2003. Preimaginal learning determines adult response to chemical stimuli in a parasitic wasp. Proceedings of the Royal Society of London. Series B: Biological Sciences 270, 2623-2629. https://doi.org/10.1098/rspb.2003.2541

Gibbs, A.G., Chippindale, A.K., Rose, M.R., 1997. Physiological mechanisms of evolved desiccation resistance in Drosophila melanogaster. J Exp Biol 200, 1821-1832. 
Gibbs, A.G., Fukuzato, F., Matzkin, L.M., 2003. Evolution of water conservation mechanisms in Drosophila. Journal of Experimental Biology 206, 1183-1192. https://doi.org/10.1242/jeb.00233

Groemping, U., 2006. Relative Importance for Linear Regression in R: The Package relaimpo. Journal of Statistical Software. https://doi.org/10.18637/jss.v017.i01

Hahn, D.A., 2005. Larval nutrition affects lipid storage and growth, but not protein or carbohydrate storage in newly eclosed adults of the grasshopper Schistocerca americana. Journal of Insect Physiology 51, 1210-1219. https://doi.org/10.1016/j.jinsphys.2005.06.011

Henry, Y., Colinet, H., 2018. Microbiota disruption leads to reduced cold tolerance in Drosophila flies. Sci Nat 105, 59. https://doi.org/10.1007/s00114-018-1584-7

Henry, Y., Renault, D., Colinet, H., 2018. Hormesis-like effect of mild larval crowding on thermotolerance in Drosophila flies (doi: 10.1242/jeb.169342). Journal of Experimental Biology 221, jeb178681. https://doi.org/10.1242/jeb.178681

Heys, C., Lizé, A., Colinet, H., Price, T.A.R., Prescott, M., Ingleby, F., Lewis, Z., 2018. Evidence That the Microbiota Counteracts Male Outbreeding Strategy by Inhibiting Sexual Signaling in Females. Front. Ecol. Evol. 6. https://doi.org/10.3389/fevo.2018.00029

Hoang, D., Kopp, A., Chandler, J.A., 2015. Interactions between Drosophila and its natural yeast symbionts-Is Saccharomyces cerevisiae a good model for studying the fly-yeast relationship? PeerJ 3, e1116. https://doi.org/10.7717/peerj.1116

Hoover, S.E.R., Higo, H.A., Winston, M.L., 2005. Worker honey bee ovary development: seasonal variation and the influence of larval and adult nutrition. J Comp Physiol B 176, 55. https://doi.org/10.1007/s00360-005-0032-0

Huang, J.-H., Douglas, A.E., 2015. Consumption of dietary sugar by gut bacteria determines Drosophila lipid content. Biology Letters 11, 20150469. https://doi.org/10.1098/rsbl.2015.0469

Hulbert, A.J., Clancy, D.J., Mair, W., Braeckman, B.P., Gems, D., Partridge, L., 2004. Metabolic rate is not reduced by dietary-restriction or by lowered insulin/IGF-1 signalling and is not correlated with individual lifespan in Drosophila melanogaster. Experimental Gerontology 39, 1137-1143. https://doi.org/10.1016/j.exger.2004.04.006

Ja, W.W., Carvalho, G.B., Mak, E.M., Rosa, N.N. de la, Fang, A.Y., Liong, J.C., Brummel, T., Benzer, S., 2007. Prandiology of Drosophila and the CAFE assay. PNAS 104, 8253-8256. https://doi.org/10.1073/pnas.0702726104

Jehrke, L., Stewart, F.A., Droste, A., Beller, M., 2018. The impact of genome variation and diet on the metabolic phenotype and microbiome composition of Drosophila melanogaster. Scientific Reports 8, 6215. https://doi.org/10.1038/s41598-018-24542-5

Jensen, P., Overgaard, J., Loeschcke, V., Schou, M.F., Malte, H., Kristensen, T.N., 2014. Inbreeding effects on standard metabolic rate investigated at cold, benign and hot temperatures in Drosophila melanogaster. Journal of Insect Physiology 62, 11-20. https://doi.org/10.1016/j.jinsphys.2014.01.003

Jørgensen, L.B., Malte, H., Overgaard, J., 2019. How to assess Drosophila heat tolerance: Unifying static and dynamic tolerance assays to predict heat distribution limits. Functional Ecology 33, 629-642. https://doi.org/10.1111/1365-2435.13279

Kapahi, P., Zid, B.M., Harper, T., Koslover, D., Sapin, V., Benzer, S., 2004. Regulation of Lifespan in Drosophila by Modulation of Genes in the TOR Signaling Pathway. Current Biology 14, 885890. https://doi.org/10.1016/j.cub.2004.03.059

Keebaugh, E.S., Yamada, R., Obadia, B., Ludington, W.B., Ja, W.W., 2018. Microbial quantity impacts Drosophila nutrition, development, and lifespan. iScience 0. https://doi.org/10.1016/j.isci.2018.06.004

Klepsatel, P., Gáliková, M., Xu, Y., Kühnlein, R.P., 2016. Thermal stress depletes energy reserves in Drosophila. Scientific Reports 6, 33667. https://doi.org/10.1038/srep33667

Kolss, M., Vijendravarma, R.K., Schwaller, G., Kawecki, T.J., 2009. Life-History Consequences of Adaptation to Larval Nutritional Stress in Drosophila. Evolution 63, 2389-2401. https://doi.org/10.1111/j.1558-5646.2009.00718.x

Koyama, T., Mendes, C.C., Mirth, C.K., 2013. Mechanisms regulating nutrition-dependent developmental plasticity through organ-specific effects in insects. Front Physiol 4. https://doi.org/10.3389/fphys.2013.00263 
Koyle, M.L., Veloz, M., Judd, A.M., Wong, A.C.-N., Newell, P.D., Douglas, A.E., Chaston, J.M., 2016. Rearing the Fruit Fly Drosophila melanogaster Under Axenic and Gnotobiotic Conditions. JoVE (Journal of Visualized Experiments) e54219-e54219. https://doi.org/10.3791/54219

Kristensen, T.N., Henningsen, A.K., Aastrup, C., Bech-Hansen, M., Bjerre, L.B.H., Carlsen, B., Hagstrup, M., Jensen, S.G., Karlsen, P., Kristensen, L., Lundsgaard, C., Møller, T., Nielsen, L.D., Starcke, C., Sørensen, C.R., Schou, M.F., 2016. Fitness components of Drosophila melanogaster developed on a standard laboratory diet or a typical natural food source. Insect Science 23, 771-779. https://doi.org/10.1111/1744-7917.12239

Layalle, S., Arquier, N., Léopold, P., 2008. The TOR Pathway Couples Nutrition and Developmental Timing in Drosophila. Developmental Cell 15, 568-577. https://doi.org/10.1016/j.devcel.2008.08.003

Le Bourg, E., Gauthier, T., Colinet, H., 2015. Feeding on frozen live yeast has some deleterious effects in Drosophila melanogaster. Experimental Gerontology 69, 202-210. https://doi.org/10.1016/j.exger.2015.06.019

Le Rohellec, M., Le Bourg, E., 2009. Contrasted effects of suppressing live yeast from food on longevity, aging and resistance to several stresses in Drosophila melanogaster. Exp. Gerontol. 44, 695-707. https://doi.org/10.1016/j.exger.2009.08.001

Lee, K.P., Jang, T., 2014. Exploring the nutritional basis of starvation resistance in Drosophila melanogaster. Functional Ecology 28, 1144-1155. https://doi.org/10.1111/1365-2435.12247

Lee, K.P., Simpson, S.J., Clissold, F.J., Brooks, R., Ballard, J.W.O., Taylor, P.W., Soran, N., Raubenheimer, D., 2008. Lifespan and reproduction in Drosophila: New insights from nutritional geometry. PNAS 105, 2498-2503. https://doi.org/10.1073/pnas.0710787105

Leitão-Gonçalves, R., Carvalho-Santos, Z., Francisco, A.P., Fioreze, G.T., Anjos, M., Baltazar, C., Elias, A.P., Itskov, P.M., Piper, M.D.W., Ribeiro, C., 2017. Commensal bacteria and essential amino acids control food choice behavior and reproduction. PLOS Biology 15, e2000862. https://doi.org/10.1371/journal.pbio.2000862

Lighton, J.R.B., Halsey, L.G., 2011. Flow-through respirometry applied to chamber systems: Pros and cons, hints and tips. Comparative Biochemistry and Physiology Part A: Molecular \& Integrative Physiology, The challenge of measuring energy expenditure: current field and laboratory methods 158, 265-275. https://doi.org/10.1016/j.cbpa.2010.11.026

Mair, W., Piper, M.D.W., Partridge, L., 2005. Calories do not explain extension of life span by dietary restriction in Drosophila. PLoS Biol. 3, e223. https://doi.org/10.1371/journal.pbio.0030223

Masoro, E.J., 2003. Caloric Restriction: A Key to Understanding and Modulating Aging, Volume 1, 1 edition. ed. Elsevier Science, Amsterdam; Boston.

Matos, R.C., Schwarzer, M., Gervais, H., Courtin, P., Joncour, P., Gillet, B., Ma, D., Bulteau, A.-L., Martino, M.E., Hughes, S., Chapot-Chartier, M.-P., Leulier, F., 2017. D-Alanylation of teichoic acids contributes to Lactobacillus plantarum -mediated Drosophila growth during chronic undernutrition. Nature Microbiology 2, 1635-1647. https://doi.org/10.1038/s41564-017-0038$\mathrm{x}$

Matzkin, L.M., Johnson, S., Paight, C., Bozinovic, G., Markow, T.A., 2011. Dietary Protein and Sugar Differentially Affect Development and Metabolic Pools in Ecologically Diverse Drosophila. J Nutr 141, 1127-1133. https://doi.org/10.3945/jn.111.138438

Min, K.-J., Flatt, T., Kulaots, I., Tatar, M., 2007. Counting calories in Drosophila diet restriction. Experimental Gerontology 42, 247-251. https://doi.org/10.1016/j.exger.2006.10.009

Moghadam, N.N., Thorshauge, P.M., Kristensen, T.N., Jonge, N. de, Bahrndorff, S., Kjeldal, H., Nielsen, J.L., 2018. Strong responses of Drosophila melanogaster microbiota to developmental temperature. Fly 12, 1-12. https://doi.org/10.1080/19336934.2017.1394558

Montllor, C.B., Maxmen, A., Purcell, A.H., 2002. Facultative bacterial endosymbionts benefit pea aphids Acyrthosiphon pisum under heat stress. Ecological Entomology 27, 189-195. https://doi.org/10.1046/j.1365-2311.2002.00393.x

Musselman, L.P., Fink, J.L., Narzinski, K., Ramachandran, P.V., Hathiramani, S.S., Cagan, R.L., Baranski, T.J., 2011. A high-sugar diet produces obesity and insulin resistance in wild-type Drosophila. Disease Models \& Mechanisms 4, 842-849. https://doi.org/10.1242/dmm.007948 
Newell, P.D., Douglas, A.E., 2014. Interspecies Interactions Determine the Impact of the Gut Microbiota on Nutrient Allocation in Drosophila melanogaster. Appl. Environ. Microbiol. 80, 788-796. https://doi.org/10.1128/AEM.02742-13

Overgaard, J., MacMillan, H.A., 2017. The Integrative Physiology of Insect Chill Tolerance. Annual Review of Physiology 79, 187-208. https://doi.org/10.1146/annurev-physiol-022516-034142

R Core Team, 2017. R: A language and environment for statistical computing. R Foundation for Statistical Computing, Vienna, Austria.

Raubenheimer, D., Simpson, S.J., Mayntz, D., 2009. Nutrition, ecology and nutritional ecology: toward an integrated framework. Functional Ecology 23, 4-16. https://doi.org/10.1111/j.13652435.2009.01522.x

Ridley, E.V., Wong, A.C.-N., Westmiller, S., Douglas, A.E., 2012. Impact of the Resident Microbiota on the Nutritional Phenotype of Drosophila melanogaster. PLOS ONE 7, e36765. https://doi.org/10.1371/journal.pone.0036765

Rion, S., Kawecki, T.J., 2007. Evolutionary biology of starvation resistance: what we have learned from Drosophila. Journal of Evolutionary Biology 20, 1655-1664. https://doi.org/10.1111/j.14209101.2007.01405.x

Rogina, B., Helfand, S.L., Frankel, S., 2002. Longevity Regulation by Drosophila Rpd3 Deacetylase and Caloric Restriction. Science 298, 1745-1745. https://doi.org/10.1126/science.1078986

Russell, J.A., Moran, N.A., 2006. Costs and benefits of symbiont infection in aphids: variation among symbionts and across temperatures. Proceedings of the Royal Society of London B: Biological Sciences 273, 603-610. https://doi.org/10.1098/rspb.2005.3348

Sannino, D.R., Dobson, A.J., Edwards, K., Angert, E.R., Buchon, N., 2018. The Drosophila melanogaster Gut Microbiota Provisions Thiamine to Its Host. mBio 9, e00155-18. https://doi.org/10.1128/mBio.00155-18

Shin, S.C., Kim, S.-H., You, H., Kim, B., Kim, A.C., Lee, K.-A., Yoon, J.-H., Ryu, J.-H., Lee, W.-J., 2011. Drosophila microbiome modulates host developmental and metabolic homeostasis via insulin signaling. Science 334, 670-674. https://doi.org/10.1126/science.1212782

Simmons, F.H., Bradley, T.J., 1997. An analysis of resource allocation in response to dietary yeast in Drosophila melanogaster. Journal of Insect Physiology 43, 779-788. https://doi.org/10.1016/S0022-1910(97)00037-1

Simpson, S.J., Raubenheimer, D., 2012. The Nature of Nutrition: A Unifying Framework from Animal Adaptation to Human Obesity. Princeton University Press.

Simpson, S.J., Sword, G.A., Lorch, P.D., Couzin, I.D., 2006. Cannibal crickets on a forced march for protein and salt. PNAS 103, 4152-4156. https://doi.org/10.1073/pnas.0508915103

Sisodia, S., Singh, B.N., 2012. Experimental Evidence for Nutrition Regulated Stress Resistance in Drosophila ananassae. PLOS ONE 7, e46131. https://doi.org/10.1371/journal.pone.0046131

Skorupa, D.A., Dervisefendic, A., Zwiener, J., Pletcher, S.D., 2008. Dietary composition specifies consumption, obesity, and lifespan in Drosophila melanogaster. Aging Cell 7, 478-490. https://doi.org/10.1111/j.1474-9726.2008.00400.x

Solon-Biet, S.M., McMahon, A.C., Ballard, J.W.O., Ruohonen, K., Wu, L.E., Cogger, V.C., Warren, A., Huang, X., Pichaud, N., Melvin, R.G., Gokarn, R., Khalil, M., Turner, N., Cooney, G.J., Sinclair, D.A., Raubenheimer, D., Le Couteur, D.G., Simpson, S.J., 2014. The Ratio of Macronutrients, Not Caloric Intake, Dictates Cardiometabolic Health, Aging, and Longevity in Ad Libitum-Fed Mice. Cell Metabolism 19, 418-430. https://doi.org/10.1016/j.cmet.2014.02.009

Storelli, G., Defaye, A., Erkosar, B., Hols, P., Royet, J., Leulier, F., 2011. Lactobacillus plantarum promotes Drosophila systemic growth by modulating hormonal signals through TOR-dependent nutrient sensing. Cell Metab. 14, 403-414. https://doi.org/10.1016/j.cmet.2011.07.012

Téfit, M.A., Gillet, B., Joncour, P., Hughes, S., Leulier, F., 2018. Stable association of a Drosophiladerived microbiota with its animal partner and the nutritional environment throughout a fly population's life cycle. Journal of Insect Physiology, SI: Nutritional Homeostasis 106, 2-12. https://doi.org/10.1016/j.jinsphys.2017.09.003

Tennessen, J.M., Barry, W.E., Cox, J., Thummel, C.S., 2014. Methods for studying metabolism in Drosophila. Methods, Drosophila developmental biology methods 68, 105-115. https://doi.org/10.1016/j.ymeth.2014.02.034 
Wong, A.C.-N., Dobson, A.J., Douglas, A.E., 2014. Gut microbiota dictates the metabolic response of Drosophila to diet. Journal of Experimental Biology 217, 1894-1901. https://doi.org/10.1242/jeb.101725

Yamada, R., Deshpande, S.A., Bruce, K.D., Mak, E.M., Ja, W.W., 2015. Microbes Promote Amino Acid Harvest to Rescue Undernutrition in Drosophila. Cell Reports 10, 865-872. https://doi.org/10.1016/j.celrep.2015.01.018

Zhu, C.-T., Ingelmo, P., Rand, D.M., 2014. G×G×E for Lifespan in Drosophila: Mitochondrial, Nuclear, and Dietary Interactions that Modify Longevity. PLOS Genetics 10, e1004354. https://doi.org/10.1371/journal.pgen.1004354 


\section{Figure captions}

714 Figure 1: Bacterial and yeast load of adult female flies. Colors show the different diets. Dots represent

715

716

717

718

719

720 replicated $\mathrm{CFU}$ countings $(\mathrm{N}=6 * 5$ flies per condition). Filled dots are for individuals with unmanipulated microbiota, and open dots for individuals with antibiotic treatment. Horizontal black lines are medians.

Figure 2: Weight and body composition of adult female flies. Colors represent the different diets. Filled dots represent measurements for individuals with unmanipulated microbiota, and open dots measurements for individuals with antibiotic treatment. In A, B and C, dots represent means for 14-21 individuals ( $\mathrm{N}=7$ per condition); in $\mathrm{D}$ and $\mathrm{E}$, dots represent pools of 5 individuals ( $\mathrm{N}=6$ per condition). Horizontal black lines are means, and error bars are $95 \%$ confidence intervals around the mean.

Figure 3: Developmental, metabolic and behavioral characteristics of adult female flies. Colors represent the different diets. Filled dots represent measurements for individuals with unmanipulated microbiota, and open dots measurements for individuals with antibiotic treatment. In A, dots are individual adult emergence events; in B and C, dots represent the mean for 14-21 individuals ( $N=7$ per condition); in D and $\mathrm{E}$, dots are the mean for 7 individuals ( $\mathrm{N}=8$ per condition). Food preference index is the subtraction of sugar consumption to yeast consumption in a choice experiment. Total consumption is the addition of sugar and yeast consumption in the same choice experiment. Horizontal black lines are means, and error bars are $95 \%$ confidence intervals around the mean.

Figure 4: Stress tolerance characteristics of adult female flies. Colors represent the different diets. Filled dots/plain lines represent measurements for individuals with unmanipulated microbiota, and open dots/dashed lines measurements for individuals with antibiotic treatment. A and B are for cold stress, C and $\mathrm{D}$ are for heat stress, $\mathrm{E}$ is for starvation, $\mathrm{F}$ is for desiccation. In $\mathrm{A}$ and $\mathrm{C}$, lines represent survival predictions of non-linear binomial model; in $\mathrm{B}$ and $\mathrm{D}$, dots represent individual coma events $(\mathrm{N}=25)$, in $\mathrm{E}$ and $\mathrm{F}$, lines and dots represent survival proportion timepoints of 25 individualized flies. Horizontal black lines in B and D represent the mean. Shades in A and error bars in B and D represent $95 \%$ confidence intervals around the mean. No confidence intervals shades are presented in $\mathrm{C}$, as they all overlapped. 


\section{Table}

742 Table 1: General table of effects of diet, microbiota, and diet*microbiota, for all measured metrics.

743

\begin{tabular}{|c|c|c|c|c|c|c|c|c|c|}
\hline & Diet & & & Microbiota & & & $t^{*}$ microbi & & \\
\hline $\begin{array}{c}\text { Chi } \\
\text { statistic } \\
(\mathrm{df}=4)\end{array}$ & $p$ value & $\begin{array}{l}\text { Variance } \\
\text { explained }\end{array}$ & $\begin{array}{c}\text { Chi } \\
\text { statistic } \\
(d f=1)\end{array}$ & $p$ value & $\begin{array}{l}\text { Variance } \\
\text { explained }\end{array}$ & $\begin{array}{c}\text { Chi } \\
\text { statistic } \\
(\mathrm{df}=4)\end{array}$ & $p$ value & $\begin{array}{l}\text { Variance } \\
\text { explained }\end{array}$ & $\begin{array}{c}\text { Variance } \\
\text { explained by } \\
\text { the model (\%) }\end{array}$ \\
\hline
\end{tabular}

\begin{tabular}{|c|c|c|c|c|c|c|c|c|c|c|}
\hline Bacterial CFU & 50,34 & $p<0.001$ & 0,25 & 117,41 & $p<0.001$ & 0,59 & 30,01 & $p<0.001$ & 0,15 & - \\
\hline Yeast CFU & 60,73 & $p<0.001$ & 0,46 & 57,07 & $p<0.001$ & 0,43 & 14,07 & $p=0.007$ & 0,11 & - \\
\hline Fresh mass & 330,17 & $p<0.001$ & 0,95 & 0,13 & $p=0.716$ & $<0.01$ & 17,36 & $p=0.002$ & 0,05 & 86,34 \\
\hline Water content & 291,27 & $p<0.001$ & 0,97 & 2,72 & $p=0.099$ & 0,01 & 4,57 & $p=0.333$ & 0,02 & 84,52 \\
\hline Total lipid & 908,72 & $p<0.001$ & 0,99 & 0,21 & $p=0.649$ & $<0.01$ & 4,23 & $\mathrm{p}=0.374$ & $<0.01$ & 94,32 \\
\hline Glucose content & 861,86 & $p<0.001$ & 0,92 & 0,01 & $p=0.913$ & $<0.01$ & 70,57 & $p<0.001$ & 0,07 & 94,91 \\
\hline Glycogen content & 146,99 & $p<0.001$ & 0,86 & 1,57 & $p=0.210$ & $<0.01$ & 21,4 & $p<0.001$ & 0,12 & 77,27 \\
\hline Development rate & 3901,09 & $p<0.001$ & 0,82 & 378,67 & $p<0.001$ & 0,08 & 446,12 & $p<0.001$ & 0,09 & 86,61 \\
\hline Dioxygen consumption & 572,49 & $p<0.001$ & 0,98 & 0,32 & $\mathrm{p}=0.571$ & $<0.01$ & 11,63 & $p=0.020$ & 0,02 & 91,4 \\
\hline $\mathrm{RQ}$ & 90,36 & $p<0.001$ & 0,95 & 0,73 & $p=0.391$ & $<0.01$ & 3,89 & $\mathrm{p}=0.421$ & 0,04 & 63,24 \\
\hline Food preference & 177,93 & $p<0.001$ & 0,94 & 3,18 & $p=0.074$ & 0,01 & 7,97 & $p=0.092$ & 0,04 & 74,03 \\
\hline Food consumption & 58,73 & $p<0.001$ & 0,88 & 0,17 & $p=0.679$ & $<0.01$ & 7,56 & $p=0.109$ & 0,11 & 50,17 \\
\hline Cold stress survival & 555,21 & $p<0.001$ & 0,96 & 17,11 & $p<0.001$ & 0,03 & 6,53 & $p=0.163$ & 0,01 & 82,46 \\
\hline Cold knockdown $t^{\circ} \mathrm{C}$ & 28,45 & $p<0.001$ & 0,11 & 149,54 & $p<0.001$ & 0,59 & 73,18 & $p<0.001$ & 0,29 & 51,31 \\
\hline Heat stress survival & 28,13 & $p=0.032$ & 0,73 & 0,02 & $\mathrm{p}=0.898$ & $<0.01$ & 10,27 & $p=0.036$ & 0,27 & 96,85 \\
\hline Heat knockdown $t^{\circ} \mathrm{C}$ & 10,85 & $p=0.028$ & 0,3 & 16,85 & $p=0.049$ & 0,47 & 7,93 & $p=0.094$ & 0,22 & 12,93 \\
\hline Starvation tolerance & 262,22 & $p<0.001$ & 0,86 & 19,36 & $p<0.001$ & 0,06 & 21,81 & $p<0.001$ & 0,07 & - \\
\hline Dessication tolerance & 29,69 & $p<0.001$ & 0,78 & 1,56 & $p=0.210$ & 0,04 & 6,8 & $p=0.146$ & 0,18 & - \\
\hline
\end{tabular}

744

745

746

747

748 


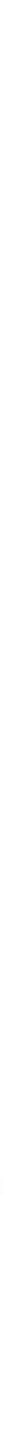




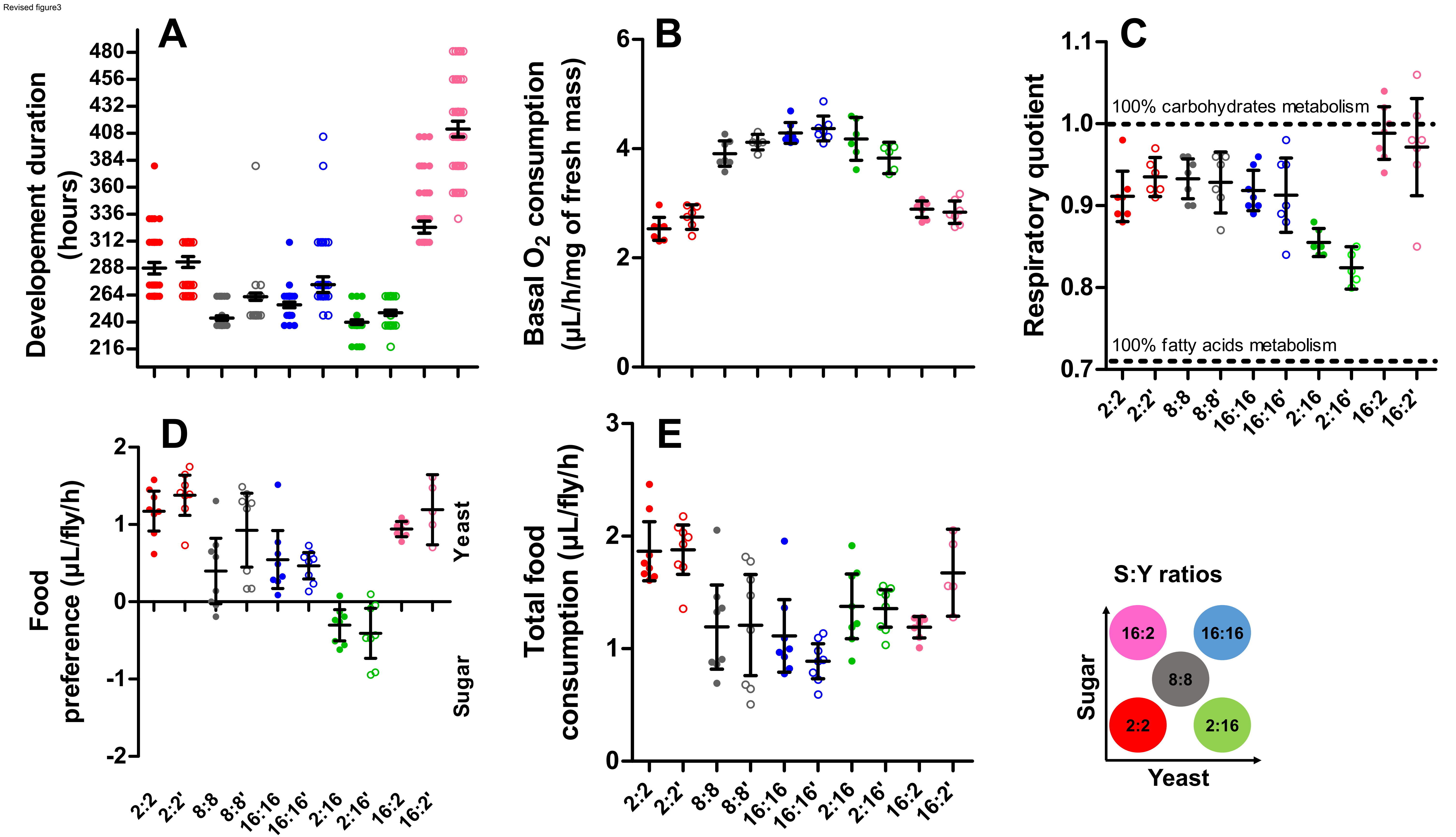


Click here to access/download
Supplementary Material
ESM.docx

Click here to access/download
Supplementary Material
ESM.docx Click here to access/download
Supplementary Material
ESM.docx

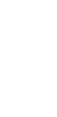

.

.
Click here to access/download
Supplementary Material
ESM.docx

Click here to access/download
Supplementary Material
ESM.docx

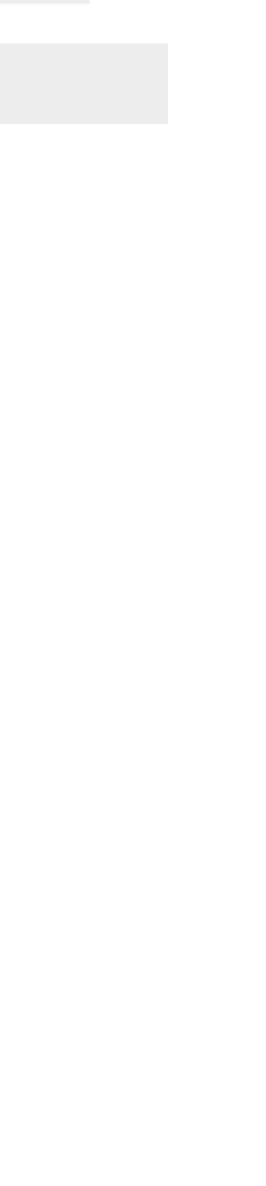

